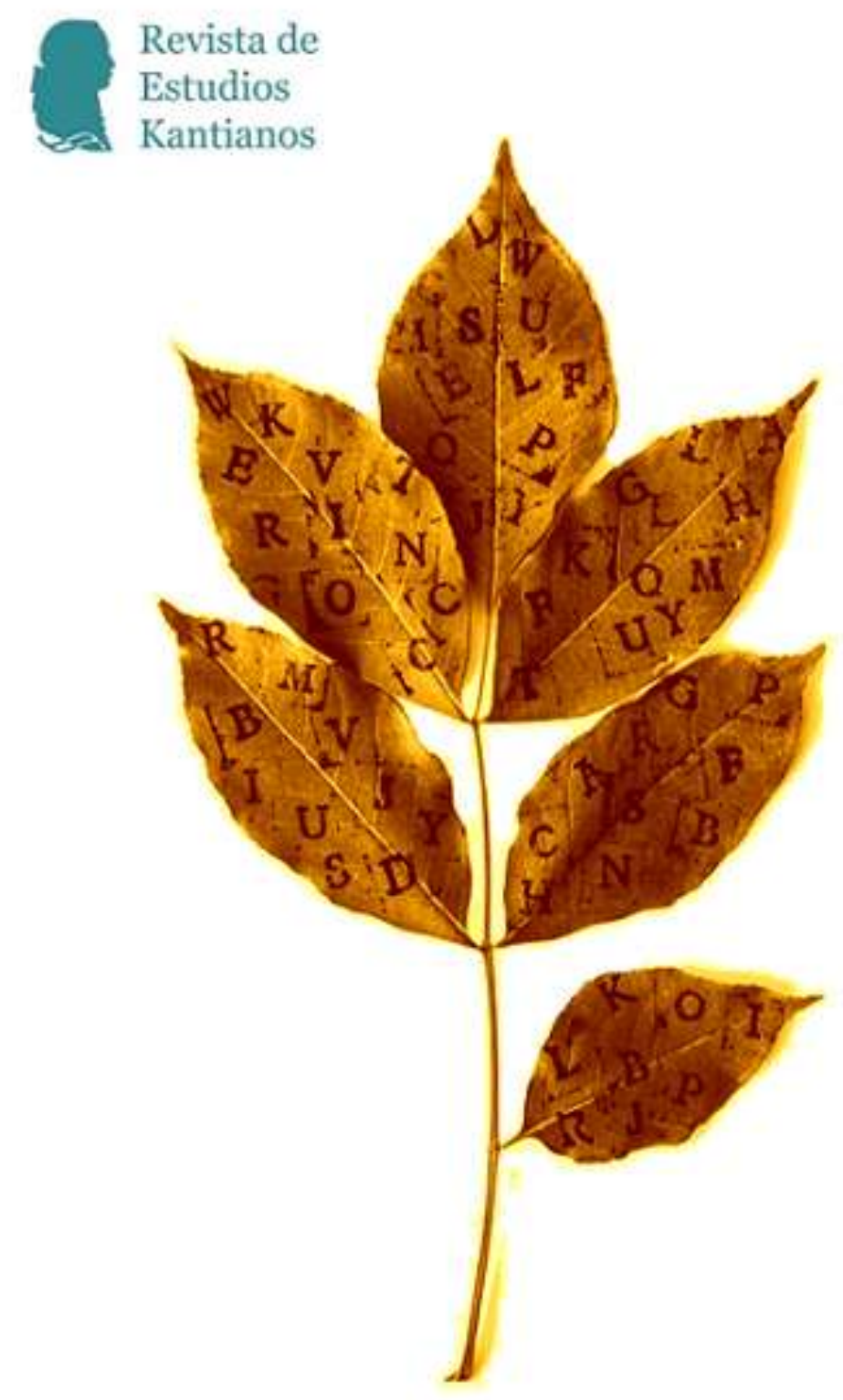




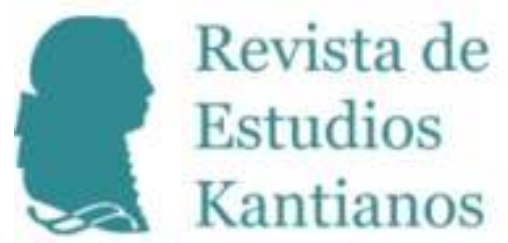




\section{Revista de Estudios Kantianos}

Publicación internacional de la Sociedad de Estudios Kantianos en Lengua Española Internationale Zeitschrift der Gesellschaft für Kant-Studien in Spanischer Sprache International Journal of the Society of Kantian Studies in the Spanish Language

\section{Dirección}

Pedro Jesús Teruel, Universitat de València

pedro.teruel@uv.es

Hernán Pringe, CONICET-Universidad de Buenos Aires/

Universidad Diego Portales, Santiago de Chile

hpringe@gmail.com

\section{Secretario de edición}

Fernando Moledo, Universidad de Buenos Aires - CONICET

fernandomoledo@gmail.com

\section{Secretaria de calidad}

Marcela García, Instituto de Investigaciones Filosóficas, UNAM

garciar.marcela@gmail.com

\section{Editores científicos}

Jacinto Rivera de Rosales, UNED, Madrid

Claudia Jáuregui, Universidad de Buenos Aires

Vicente Durán, Pontificia Universidad Javeriana, Bogotá

Julio del Valle, Pontificia Universidad Católica del Perú, Lima

Jesús Conill, Universitat de València

Gustavo Leyva, Universidad Autónoma de México, México D. F.

María Xesús Vázquez Lobeiras, Universidade de Santiago de Compostela

Wilson Herrera, Universidad del Rosario, Bogotá

Pablo Oyarzun, Universidad de Chile, Santiago de Chile

Paula Órdenes Azúa, Universität Heidelberg 


\section{Comité científico}

Juan Arana, Universidad de Sevilla

Reinhardt Brandt, Philipps-Universität Marburg

Mario Caimi, Universidad de Buenos Aires

Monique Castillo, Université de Paris-Est

Adela Cortina, Universitat de València

Bernd Dörflinger, Universität Trier

Norbert Fischer, Universität Eichstätt-Ingolstadt

Miguel Giusti, Pontificia Universidad Católica del Perú

Dulce María Granja, Universidad Nacional Autónoma de México

Christian Hamm, Universidad Federal de Santa María, Brasil

Dietmar Heidemann, Université du Luxembourg

Otfried Höffe, Universität Tübingen

Claudio La Rocca, Università degli Studi di Genova

Juan Manuel Navarro Cordón, Universidad Complutense, Madrid

Carlos Pereda, Universidad Nacional Autónoma de México

Gustavo Pereira, Universidad de la República, Uruguay

Ubirajara Rancan de Azevedo, Universidade Estadual Paulista, Brasil

Margit Ruffing, Johannes Gutenberg-Universität Mainz

Gustavo Sarmiento, Universidad Simón Bolívar, Venezuela

Sergio Sevilla, Universitat de València

Roberto Torretti, Universidad Diego Portales, Santiago de Chile

Violetta Waibel, Universität Wien

Howard Williams, University of Aberystwyth

Allen W. Wood, Indiana University

Diseño, revisión de estilo y maqueta

Josefa Ros Velasco, Universidad Complutense de Madrid

\section{Entidades colaboradoras}

Sociedad de Estudios Kantianos en Lengua Española (SEKLE)

Departament de Filosofia de la Universitat de València 




Artículos

113 ¿"El yo propiamente tal” (I. Kant) o "el originario ser sí mismo" (D. Heinrich)? Sobre algunas características del concepto kantiano de autoconciencia

Heiner Klemme

DOI 10.7203/REK.2.2.9068

126 La noción kantiana de verdad transcendental

Stéfano Straulino Torre

DOI 10.7203/REK.2.2.8809

146 Una travesía kantiana a través del Escila constructivista y el Caribdis realista. Apuntes para un abordaje kantiano-constitutivista de las fuentes de la normatividad

Martín Fleitas González.

DOI 10.7203/REK.2.2.8810

174 El malestar kantiano. Filosofía y ciencia al encuentro con la naturaleza Juan Felipe Guevara

DOI 10.7203/REK.2.2.8812

191 ¿Puede haber una fundamentación kantiana de los derechos humanos? Algunas consideraciones críticas

Nuria Sánchez Madrid

DOI 10.7203/REK.2.2.8871

\section{Semblanza}

208 El pensamiento filosófico de Ernesto Mayz Vallenilla

Gustavo Sarmiento

DOI 10.7203/REK.2.2.9021 


\section{Reseñas}

217 Claudia Jáuregi, Fernando Moledo, Hernán Pringe y Marcos Thisted (eds.) Crítica y Metafísica. Homenaje a Mario Caimi. Studien und Materialen zur Geschichten der Philosophie. Hildesheim, Olms, 2015, 460 pp. ISBN: 978-3-487-15237-0.

Alba Jiménez Rodríguez.

DOI 10.7203/REK.2.2. 8814

227 Fernando Moledo: Los años silenciosos de Kant: Aspectos de la génesis de la Deducción Trascendental en la década de 1770. Seguido de la traducción del Legado de Duisburg (ca. 1775). Buenos Aires, Prometeo, 2014, 192 pp. ISBN: 978-987-574-667-1.

Pablo Moscón DOI 10.7203/REK.2.2. 8830

231 Salvi Turró: Filosofia i Modernitat. La reconstrucció de l'ordre del món. Barcelona, Edicions Universitat de Barcelona, 2016, 228 pp. ISBN: 978-84-475-3966-6.

Pedro Jesús Teruel

DOI 10.7203/REK.2.2. 8887

233 Gabriel Rivero: Zur Bedeutung des Begriffs Ontologie bei Kant. Berlín, Boston, Walter de Gruyter, 2014, 247 pp. ISBN: 978-3-11-034180-5.

Luciana Martínez

DOI 10.7203/REK.2.2.8881

Informe

237 Informe sobre el III Congreso de la Sociedad de Estudios Kantianos en Lengua Española (SEKLE)

Luciana Martínez

DOI 10.7203/REK.2.2.9011

\section{Convocatoria}

242 La recepción de Kant en México

Dulce María Granja Castro; Gustavo Leyva Martínez

Eventos y normas para la publicación

244 Noticias y normas para autores 


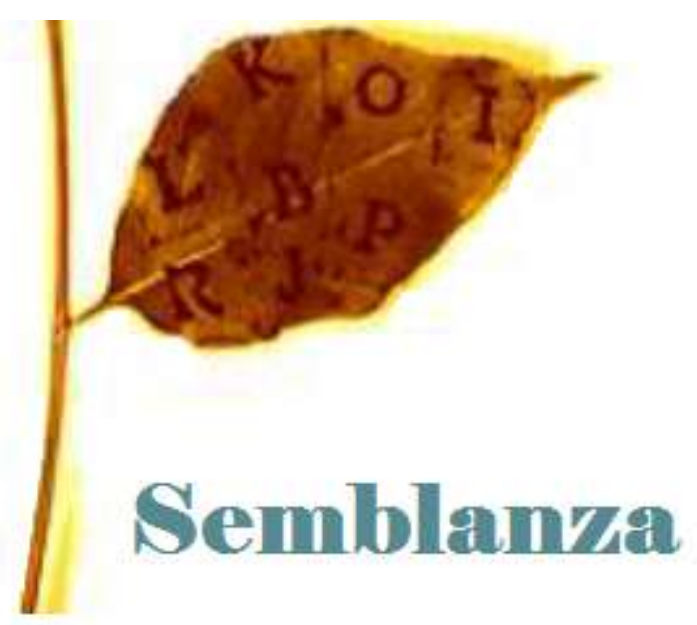




\title{
El pensamiento filosófico de Ernesto Mayz Vallenilla
}

\author{
GuSTAVO AlbERTo SARMIENTO ${ }^{1}$
}

\begin{abstract}
Nacido en Maracaibo el 3 de septiembre de 1925 y fallecido en Caracas el 25 de diciembre de 2015, Ernesto Mayz Vallenilla fue uno de los venezolanos más destacados de este tiempo. Maestro de varias generaciones de intelectuales, las instruyó en problemas y autores principales de la filosofía, así como enseñó a discípulos y alumnos los métodos del pensamiento riguroso, estimulando una pléyade de vocaciones. Autor de valiosos trabajos hermenéuticos sobre temas y pensadores de la filosofía occidental, Mayz Vallenilla se movió, de modo inusual en nuestro medio, más allá de la interpretación filosófica hacia un pensamiento propio, el cual encuentra su mayor realización en su obra capital, los Fundamentos de la meta-técnica, cuya primera edición data de 1990. Como maestro, intérprete y filósofo original, Ernesto Mayz Vallenilla dio una contribución primordial a la normalización de la actividad filosófica en Venezuela.
\end{abstract}

En tanto hombre de acción, obró en el progreso o en la misma forja de muchas instituciones académicas. Mencionemos aquí a la Universidad Central de Venezuela, la Universidad Simón Bolivar, el Instituto de Estudios Avanzados (IDEA) o la Sociedad Venezolana de Filosofía. En la Universidad Central de Venezuela, su alma mater, Mayz llevó a cabo una dilatada labor docente y su entusiasmo se hizo sentir de diversas maneras en la Escuela de Filosofía, en el Instituto de Filosofía y en instancias más elevadas, como la Comisión Universitaria nombrada a la caída de la dictadura de Marcos Pérez Jiménez. El trabajo fundacional de Mayz en la Universidad Simón Bolívar de Caracas es tal vez la causa primordial de la calidad que ha tenido esa institución, la cual todavía conserva el modo de ser en ella sembrado por él. En IDEA, este maestro dirigió la unidad de filosofía, cobijando el desarrollo de las carreras de jóvenes investigadores quienes hoy en día dan clases en universidades venezolanas y de otros países.

Logros sobresalientes como estos revelan una voluntad decidida. En el hombre, la voluntad obra a partir de la representación de aquello cuya existencia este quiere, moviéndolo a efectuar las acciones que han de favorecer o producir la realidad de lo que al inicio era sólo representación. Para poder ser eficiente, el querer, la voluntad, tiene que contar con recursos en los cuales apoyarse. En Ernesto Mayz Vallenilla destacaron sus innegables dotes de pensador y escritor, su inteligencia y originalidad. En su carácter hubo firmeza, pero igualmente generosidad, hubo decisión y fortaleza, entusiasmo y humor, autoconciencia y también, por qué no decirlo, ambición. No se puede hacer cosas importantes sin ambición -más aún: hay que cuidarse de quienes nada ambicionan-. Pido que se me entienda bien, la ambición a la que me refiero se justifica cuando los logros, importantes como sean, no lo son para la persona, sino para la comunidad. Es esta, tal vez, la ambición que hace a quien trabaja por el bien de las instituciones. Por otra parte, el agudo sentido práctico de Ernesto Mayz Vallenilla no es común en quienes se dedican a los estudios teóricos. Entre estos, no muchos, más bien pocos, tienen dotes para la acción, condición necesaria de una praxis eficiente. En aquellos que las poseen, el estudio de la filosofía puede ser notoriamente útil para orientar y lograr un actuar productivo. Tal es, me parece, el caso de Mayz Vallenilla, incluso se podría decir que por ser filósofo fue tan eficiente como constructor de instituciones.

Siendo su obra extensa y abarcadora de diversos temas y preocupaciones, exponerla plantea un reto difícil de cumplir a cabalidad. Con todo, intentaré presentar sus principales trabajos y contribuciones, en unos casos de manera general y en otro con más detalle.

\footnotetext{
${ }^{1}$ Universidad Simón Bolivar.
} 
La Idea de la Estructura Psíquica en Dilthey fue, en 1949, el primer libro publicado por Mayz. Más adelante, en su tesis doctoral, titulada Fenomenología del Conocimiento, publicada en 1956 y reeditada en 1976, abordó el problema de la constitución del objeto real en el campo de la conciencia. Este trabajo es una importante obra de consulta para quien quiera aproximarse al estudio e interpretación de la problemática de la constitución del objeto en la fenomenología de Edmund Husserl. En La Ontología del Conocimiento de 1960 encontramos una investigación ontológicaexistenciaria del conocimiento basada en el pensamiento de Martin Heidegger.

En 1965 apareció El Problema de la Nada en Kant. En este libro, Mayz Vallenilla toma como punto de referencia para centrar su análisis el tratamiento de la noción de la nada que se encuentra en la Crítica de la Razón Pura. El Problema de la Nada en Kant constituye, sin duda, una interpretación original de este tema, en la cual se intenta descubrir a la nada en su 'originariedad' absoluta. Sin embargo, la tentativa de pensar la nada a partir de Kant produce una tensión entre el pensamiento del intérprete y el del autor, la cual surge de la oposición en que se encuentran la concepción de la nada como algo originario y el punto de vista de la misma como algo en cierto modo derivado. Kant no consideró a la nada como originaria, de manera que el intento de Mayz Vallenilla sólo puede tener éxito si sale de los límites del pensamiento de Kant. Para ello toma como precedentes ciertos aspectos del pensamiento heideggeriano. Sin embargo, él no piensa a la nada de la misma manera que Heidegger, como ya lo sugiere el hecho de que toma como punto de partida el análisis kantiano. Al hacer esto, también busca ir más allá de los horizontes del pensamiento de Heidegger. Mayz Vallenilla intenta pensar a la nada desde el tiempo, a fin de revelar la 'temporalidad' propia de la 'nada', a la cual considera diferente de la del 'ser'. Para fundamentar esta tentativa se apoya en el marco de la reflexión kantiana (en particular la doctrina de los esquemas), tratando de aclarar aquellos puntos de la misma susceptibles de crítica, con la finalidad de analizar a partir de ella el 'esquematismo' - diferente al de las categorías positivas- que los conceptos kantianos de la nada han de tener, y a partir del cual se podrá aprehender la conexión entre el 'tiempo' propio de la nada y la 'nada' misma. Con esto quiere comprender al tiempo desde el horizonte de la propia nada y a partir de la zona fronteriza entre ella y los entes donde, a la luz de la 'diferencia ontológica', la nada logra manifestar su 'negatividad positiva, originaria y absoluta' (Mayz Vallenilla 1965: 45).

Mayz está consciente de las dificultades que salen al paso del intento de pensar la 'nada' (Mayz Vallenilla 1965: 13 ss). La principal proviene del lenguaje, pues este contiene de manera implícita una serie de categorías cuyo supuesto ontológico es el ser. El lenguaje se funda en una comprensión del ser, que, sin embargo, no es satisfactoria ya que en ella no prevalece la 'diferencia ontológica', lo cual ha mostrado Heidegger (Mayz Vallenilla 1965: 16-17). Surge entonces la duda de si el lenguaje es realmente el instrumento idóneo para expresar una noción de la nada que pone en cuestión los propios supuestos ontológicos del lenguaje. Otra dificultad para la expresión de la nada reside en la manera en que ésta ha sido pensada en el pasado (Mayz Vallenilla 1965: 32). Mayz Vallenilla señala que la tradición, ya desde los griegos, se ha aproximado a la nada a partir del ser, como negación del mismo. La nada ha sido pensada en relación con conceptos como 'comienzo, origen, fundamento', etc., tratados por la tradición a partir de conceptos del ser como los de 'causa, potencia, acto', etc., a los cuales se añade una negación, con la finalidad de transformar al ser (o su respectiva determinación) en un no-ser. Pero esta manera de comprender la nada es insatisfactoria, pues el resultado de ella es que la nada no es aprehendida en su originariedad, sino que su aprehensión es entorpecida y desnaturalizada por el empleo subrepticio de los conceptos del ser. La comprensión de la nada que así resulta es parcial y dependiente de la del ser, pues la negación -o la privación- es meramente negación de algo. De esta manera, la nada es dada solamente con relación a lo que es. Para encontrar a la nada en sí misma hay que hacer algo más que negar o suprimir el ser. Por ello, el problema fundamental para la investigación de Mayz Vallenilla es descubrir y aclarar una vía que permita aprehender a la nada en cuanto tal (Mayz Vallenilla 1965: 35). Aquí se vuelve importante el pensamiento de Heidegger, que ha puesto de relieve al tiempo como horizonte de sentido del ser. Apoyándose en este precedente, Mayz trata de pensar a la nada desde la perspectiva del tiempo, en tanto horizonte de la misma. De esta manera, la principal cuestión que estudia es la 'temporalidad de la nada'.

Para Mayz, las nociones kantianas de la nada tienen especial significado en el intento de aclarar la conexión entre el tiempo y la nada, pues ellas están ligadas a los cuatro grupos de 
categorías, a las cuales corresponde un esquematismo que les da su sentido temporal (Mayz Vallenilla 1965: 43-44). Es por ello que examina los conceptos de la nada expuestos en la KrV. Sin embargo, el esquematismo de estos conceptos no puede ser el mismo expuesto por Kant para la aplicación de las categorías a las intuiciones. La temporariedad originaria de la nada indagada por Mayz Vallenilla habrá de consistir más bien en un esquematismo puramente negativo, de signo contrario al de Kant, y por lo tanto de un 'sentido' temporal y estructura diferentes. Teniendo esto en cuenta, para arribar a la temporalidad de la nada, Mayz Vallenilla se ocupa primero del ens rationis (Mayz Vallenilla 1965: 51 ss).

La primera determinación que Kant adscribe a la noción de la nada es la de ser semejante a un 'noúmeno' (ens rationis). En cuanto tal, la nada es un concepto al cual no corresponde ninguna intuición sensible; por esto se trata de un concepto vacío sin objeto. Debido a eso, la nada no puede ser contada entre las posibilidades. Pero tampoco es algo imposible, ya que ella asume la apariencia de un mero ente de razón, al igual que el noúmeno. De acuerdo con su intento de mostrar la nada en su negatividad positiva, originaria y absoluta, a Mayz le interesa demostrar y explicar la posibilidad de que la nada sea producto de la actividad aniquiladora de la 'categoría' de ninguno sobre las intuiciones. Si esto es así, se requiere de un esquema productor de la negatividad a partir de la susodicha 'categoría' de ninguno, el cual haga posible su aplicación a las intuiciones, si bien para suprimirlas (Mayz Vallenilla 1965: 62-63). En consecuencia, ha de haber un horizonte temporal que defina su sentido. Al abordar la demostración y explicación de la posibilidad de que la nada sea producto de una actividad 'categorial' aniquiladora del concepto de ninguno, Mayz Vallenilla aspira a poder comprender, más allá del pensamiento expreso de Kant, por qué el filósofo de Königsberg creyó poder equiparar a la nada con un noúmeno, y hacer transparente cómo la nada, teniendo una 'realidad objetiva' negativa, posee también una estructura temporal arraigada en el sentido del propio esquematismo, que posibilita la síntesis de la intuición con la correspondiente categoría (Mayz Vallenilla 1965: 66-67).

De acuerdo con la $\mathrm{KrV}$ el esquema puro de las magnitudes positivas es el 'número', que consiste en la adición sucesiva de unidades homogéneas en el tiempo ( $\mathrm{KrV}$ A 142-3/B 182). De acuerdo con Mayz Vallenilla, el número negativo también expresa una condición temporal, pero el tiempo que ha de estar a la base del esquema de la magnitud negativa debe tener un horizonte "ontológico" determinado por la negatividad de la nada, y por lo tanto diferente al del tiempo corriente perteneciente a la cantidad positiva. Ese tiempo se comprende desde la nada y sus esquemas la hacen patente (Mayz Vallenilla 1965: 66-70 ss). Cuando el concepto de 'ninguno' es referido a lo múltiple dado en la intuición, su esquema temporal suprime lo positivo, produciendo su opuesto, una realidad negativa que se enfrenta a la realidad positiva de la intuición y da lugar al surgimiento de una nada que es representada numéricamente con el cero. Tal es la función que ejerce dicho concepto a través de su esquema. Lo hasta aquí dicho indica que la nada en cuanto noúmeno tiene una temporalidad característica.

En tanto función meramente inteligible de la razón pura, la apercepción trascendental parece exhibir una evidente atemporalidad. En el mismo sentido, también el noúmeno parece ser atemporal. Sin embargo, Mayz Vallenilla no cree que el 'yo trascendental' sea atemporal, sino que no exhibe las mismas características temporales del yo empírico y real. La determinación que lo caracteriza como permanente no define una 'ausencia absoluta' de tiempo, sino que expresa su 'presencia absoluta', que en cuanto presencia es temporal, en medio del flujo cambiante de la conciencia empírica (Mayz Vallenilla 1965: 83). Estos razonamientos intentan confirmar la temporalidad del 'yo' y por lo tanto de la 'apercepción trascendental', lo cual tiene consecuencias importantes para el problema del noúmeno y la nada concebida en cuanto noúmeno. En este momento es importante señalar que Mayz Vallenilla no está tratando de identificar sin más al noúmeno con la 'apercepción trascendental' o la razón pura. Al insistir en la temporalidad de la razón y de la apercepción trascendental, lo que él quiere destacar es que si bien las mismas son estructuras meramente inteligibles, no son atemporales. En esta línea de pensamiento, también al noúmeno habría que imputarle una temporalidad (Mayz Vallenilla 1965: 85).

Para Kant, el nihil privativum es el objeto vacío de un 'concepto'. La noción kantiana de la nada como nihil privativum proviene de una privación, de la cual resulta la carencia o ausencia de 
cualidades positivas en el objeto. En la $\mathrm{KrV}$, a las categorías de la cualidad no puede corresponder una negación absoluta en el campo fenoménico. En este punto Mayz Vallenilla se separa de Kant (Mayz Vallenilla 1965: 96 ss), en tanto somete a crítica el presupuesto según el cual los conceptos a priori de la 'cualidad' son 'categorías ontológicas'. Lo que él busca es mostrar que la negación es también una 'categoría de la nada' (Mayz Vallenilla 1965: 101). Mayz Vallenilla intenta comprender a la nada en sí misma y por sí misma, y no a partir del ser; en consecuencia, tiene que pensarla como negatividad absoluta y no condicionada por el ser (como algo que resulta de la negación de las cualidades positivas del ser o de los entes). Pero en la $\mathrm{KrV}$ no se concibe a la nada de esta manera, por lo cual el fin perseguido por Mayz hace fuerza al pensamiento kantiano y lo obliga a ir más allá del mismo. La clave de esto es mostrar la existencia de 'cualidades negativas' y acotar su sentido (Mayz Vallenilla 1965: 107 ss). Mas esas cualidades negativas no pueden consistir en sensaciones disminuidas, pues la negatividad buscada por Mayz es la del vacío absoluto de toda sensación. Dentro de la concepción kantiana es imposible aprehender este vacío de sensaciones, por lo cual Mayz Vallenilla se apoya en ciertas situaciones en las cuales es posible experimentar directamente un vacío de sensaciones. La primera de estas situaciones es el sueño profundo: el vacío de sensaciones del sueño no revela una ausencia de cualidades positivas, sino la presencia de 'cualidades' de la propia nada. También en la vigilia existen fenómenos que dan fe de la presencia de la nada. Tal es, nos dice Mayz Vallenilla, la experiencia de la 'angustia', descrita existenciariamente por Heidegger ${ }^{2}$, en la cual se hace patente la nada (Mayz Vallenilla 1965: 111 ss). Dicha patencia ocurre porque a la existencia le es dada la presencia de la nada. Mayz Vallenilla se refiere al fenómeno del 'anonadamiento' (die Nichtung), posibilitado por la angustia, y en el cual la nada se vuelve patente como lo absolutamente 'otro' y opuesto a lo que 'es'. Con esto Mayz Vallenilla ha puesto de relieve a la nada como 'fenómeno', pero queda por indagar cual es la estructura de semejante 'fenómeno', y para ello hay que acotar la temporalidad de la nada.

En El Problema de la Nada en Kant se delimitan dos sentidos de la negación. El primer sentido le corresponde en tanto 'negación ontológica', mientras que el segundo le pertenece como 'negación absoluta' (Mayz Vallenilla 1965: 114). Mayz Vallenilla afirma que la temporalidad es el sentido de ambas nociones, de manera que es necesario elucidar la estructura de la misma, y esto conduce al problema del esquematismo (Mayz Vallenilla 1965: 114). ¿Cuál ha de ser el esquematismo que corresponda a la negatividad absoluta, si es que ella es aplicable a los fenómenos? Desde la perspectiva de la comprensión natural del ser, el tiempo es un ente entre los entes, que se presenta como un ente 'a la mano' y 'ante los ojos', y se muestra como una sucesión de instantes donde se dan los demás entes. Pero esto no es lo que acontece cuando se manifiesta la nada (Mayz Vallenilla 1965: 125). En las formas de revelarse la nada estudiadas por Mayz Vallenilla, el anonadamiento y el sueño profundo, se da una especie de pérdida o desvanecimiento del modo real y cotidiano del tiempo, pero con ello no desaparece el tiempo (Mayz Vallenilla 1965: 129). El anonadamiento o el sueño profundo se dan también en el tiempo. No implican su desaparición absoluta, pero si hacen desaparecer su aspecto instrumental, propiciando la aparición de otro aspecto del tiempo, en el cual hay un 'vacío' de 'contenidos reales' y surge una 'presencia' de 'contenidos negativos' (Mayz Vallenilla 1965: 126-127). Tales contenidos constituyen, de acuerdo con Mayz, la 'abseidad', la nada en su pura negatividad. Así pues, el tiempo del anonadamiento o del sueño profundo no presenta nada, o mejor: 'presenta' la nada.

En tanto ens imaginarium, Kant explicaba a la nada como una 'intuición vacía sin objeto'. Por su parte, al tratar al ens imaginarium, Mayz Vallenilla busca primero esclarecer la condición del espacio y el tiempo en tanto 'imágenes puras', después trata de precisar la función del tiempo (en cuanto 'sentido interno') en su papel de esquema, y finalmente intenta establecer su función, como esquema, en relación con la nada como 'imagen pura' (Mayz Vallenilla 1965: 146). Mayz ya había indicado que había que avizorar a la nada sobre la base de la 'negatividad absoluta' (dicha tesis es

\footnotetext{
${ }^{2}$ Vid. Was ist Metaphysik?, 111 ss.

${ }^{3}$ En el sueño profundo y el anonadamiento tenemos la 'experiencia' de la posibilidad de la inexistencia del mundo real, del Yo y de todo posible correlato de la conciencia; inexistencia que revela a la nada sin ir contra el ser. En virtud de esa 'experiencia', el ser se muestra como lo que es, a la par que se vislumbran las "evanescentes fronteras de la abseidad" (Mayz Vallenilla 1965: 110) El concepto de 'abseidad' es originario de Mayz Vallenilla y no hay que confundirlo con el significado del término "aseidad", que la tradición ha entendido, de manera diferente, como la existencia por sí mismo, o por necesidad de su propia naturaleza, de un ente (Dios).
} 
central para El Problema de la Nada en Kant, Mayz Vallenilla 1965: II, 91 ss). Esta es la única manera de poder exhibir su estructura temporal y de aprehender un tiempo que, sin dejar de ser una 'imagen pura', pueda mostrar la patencia de la propia nada y no la de una mera privación del ser. El camino para ello es, en un primer esbozo, abolir las categorías ontológicas, con su esquematismo correspondiente, e indagar y deducir trascendentalmente la posibilidad de las categorías de la nada y su respectivo esquematismo (Mayz Vallenilla 1965: 195).

Kant caracteriza a la nada en tanto nihil negativum como un 'objeto vacío sin concepto', que se distingue del ens rationis porque, a diferencia de aquel, es opuesto a la posibilidad lógica, al ser el objeto de un concepto que se contradice a sí mismo. La nada del nihil negativum surge de vulnerar el principio de no-contradicción. Lo imposible es nada porque dicho imposible es auto contradictorio. Frente al punto de vista kantiano, en El Problema de la Nada en Kant Mayz Vallenilla trata de mostrar que el nihil negativum no es una nada meramente lógica, sino que la imposibilidad que expresa es también una imposibilidad real (Mayz Vallenilla 1965: 202-206). Si Mayz Vallenilla tiene razón, ha transformado la noción de posibilidad, que ahora tendría una índole ontológica, a la cual se refiere originariamente el nihil negativum. Este es imposible lógicamente en tanto es fundamentalmente imposible ontológicamente (Mayz Vallenilla 1965: 219).

Por otra parte, de acuerdo con Mayz Vallenilla, un supuesto fundamental de la doctrina de Kant es que ser y tiempo se suponen e implican mutuamente (Mayz Vallenilla 1965: 253-254). Entre ambos prevalece un círculo de recíproca irradiación, a partir del cual se establecen el sentido ontológico-temporal de lo posible y lo imposible, y el significado de los Postulados del Pensamiento Empírico en su relación con el esquematismo (Mayz Vallenilla 1965: 252). Sin embargo, frente a esta perspectiva, Mayz Vallenilla trata de mostrar la posibilidad de que a partir de la "experiencia" radical de la nada pueda hacerse patente una 'temporalidad' de signo diferente, no ontológico, que actúa como sentido del no-ser. Dicha 'temporalidad' debe originarse en la comprensión de la nada, tal como se revela en fenómenos como la angustia y el sueño profundo, y por ello no habrá de estar estructurada mediante 'ahoras' o 'instantes', como la temporalidad ontológica. El 'tiempo de la nada' es 'un tiempo sin presencias', que revela una 'ausencia', la cual es la patencia de la nada. No se trata de la desaparición del tiempo ontológico, sino de una temporalidad peculiar. Así pues, la patencia de la nada no se da en ausencia del tiempo, sino que en la misma "'hay' tiempo, así como 'hay' Nada" (Mayz Vallenilla 1965: 255). Pero ese tiempo presenta una estructura muy diferente a la del tiempo ontológico. Ahora bien, Mayz Vallenilla afirma que en el ámbito de la pura razón, abolido el nexo con los fenómenos y por lo tanto con las condiciones que limitan la posibilidad de la experiencia, lo imposible desde el punto de vista empírico se convierte en lo posible trascendental, ens extramundanum, que es sinónimo de algo imposible, nihil negativum, cuya negatividad no indica tan sólo una negación ontológica, sino que muestra la positiva negatividad de la 'razón' (Mayz Vallenilla 1965: 263-264). Lo posible trascendental así manifiesto tiene una 'posibilidad' que va más allá de la sola posibilidad ontológica y cuyo 'ser' trasciende el mundo fenoménico. Con ello, al lado del 'logos' de la experiencia se exhibe un 'logos' de la pura razón, distinto y opuesto al primero. Este 'logos' permite trascender - pero no transgredir- las leyes del mundo sensible, en tanto descubre la faz de lo inteligible en su positiva negatividad (Mayz Vallenilla 1965: 265). Esa negatividad pura, manifestada por la razón pura, testimonia que la nada, entendida como distinta y opuesta al no-ser, se hace patente en ella. De esta manera, la 'temporalidad' de la nada rebasa y trasciende a la temporalidad meramente ontológica -entendida como horizonte de sentido del ser y del no-ser-. ${ }^{4}$ Finalmente, solo cuando se note la patencia de la 'imposibilidad absoluta' - contrapuesta a la imposibilidad ontológica- puede presentarse la nada, junto con la 'temporalidad' que le da 'sentido' en el dominio de la negatividad absoluta. Esa nada ya no puede ser vista como un no-ser, mera negación del ser, sino como la esencial 'otredad' del ser.

Con esto concluyo esta presentación de las principales tesis expuestas por Ernesto Mayz Vallenilla en El Problema de la Nada en Kant, obra que se caracteriza porque su autor trata de comprender la nada en su originariedad absoluta, independiente del ser. Como tal, El Problema de la

4 "Constituyendo la negatividad absoluta de la Razón una dimensión que rehúsa por principio la legalidad ontológica, el "sentido" de su peculiar "Temporalidad" debe buscarse a partir de una "comprensión" arraigada en la dimensión que inaugura la propia Nada como ens extramundanum. De tal manera, el reino de la pura Razón no es a-temporal, sino que revela una "Temporalidad" de signo diferente a la que da y cobra sentido a partir del mundo fenoménico" (Mayz Vallenilla 1965: 266). 
Nada en Kant representa un intento original de mostrar un camino hacia la aprehensión de la nada, el cual no es directo, porque la herramienta de la investigación filosófica, el pensar y los conceptos con los cuales el mismo opera, tienen como horizonte de referencia al ser o al ente en cuanto tales, lo cual conduce a una extensa reflexión acerca del pensar, sus supuestos y sus limitaciones. Así, Mayz Vallenilla afirma que en la intelección de lo imposible, el nihil negativum en tanto ens extramundanum, el pensar ontológico fracasa por una imposibilidad esencial para "encararse -desde los supuestos que lo sostienen y nutren - con un problema que desborda y niega sus propias bases" (Mayz Vallenilla 1965: 267). Sin embargo, desde dicho fracaso y el conocimiento de su razón, es posible avizorar la nada, aunque ésta no sea aprehensible ni explicable a partir de dicho pensar.

El problema de la educación, particularmente el de la universidad y la validez que esta institución pueda conservar en el mundo contemporáneo es otro de los temas que Mayz Vallenilla ha considerado central y al cual ha aplicado su pensamiento. Son muchos los escritos en los cuales se ocupó de este asunto durante medio siglo. Entre los mismos es fundamental El Ocaso de las Universidades, aparecido en 1984, trabajo en el cual, partiendo de una meditación acerca de la ratio technica y sus efectos en la organización de la universidad contemporánea, Mayz propone una superación de la noción tradicional de universidad y de los fundamentos de la misma. El Ocaso de las Universidades es una reflexión acerca de los fundamentos que articulan a la universidad, con el objeto de resaltar las condiciones necesarias para una reforma de esta institución. Mayz Vallenilla pone de relieve el mayor problema de los sistemas universitarios contemporáneos y en particular del venezolano, el cual consiste en que las instancias del mundo actual no son aprehendidas en ellos y se desconoce el gran desarrollo alcanzado por la ciencia y la técnica. Para Mayz, la misión más importante de la universidad estriba en develar al hombre el complejo y articulado mundo en el cual ha de insertar su propia acción. Se trata de constituir un conocimiento teórico no disociado de la práctica. Siguiendo este proyecto, en la universidad se ha de forjar una idea del mundo para que la acción humana encuentre las mejores condiciones de inserción. El diseño de reforma del sistema educativo propuesto por Mayz Vallenilla debe ser enmarcado dentro de esta perspectiva. La misma es una reforma que busca remediar el sueño quebrantado de una institución autosuficiente, autónoma y democrática, asfixiada por una progresiva burocratización y por el control de los partidos políticos. De no llevarse a cabo esta tarea, la universidad quedaría marginada, condenándose a su propio ocaso.

Otra preocupación de Ernesto Mayz Vallenilla fue la de las condiciones para la elaboración de un pensamiento filosófico original de América Latina. Aquí es fundamental El Problema de América de 1959. Como respuesta al problema de la falta de originalidad del pensamiento latinoamericano, Mayz Vallenilla sostiene que el único recurso al que pueden recurrir los latinoamericanos para ser originales y originarios es entregarse a vivir lo más auténticamente posible su propio modo de ser. Ese modo de ser es el de 'habitantes de un Nuevo Mundo'. Ahora bien, alcanzar la originariedad del propio ser como hombres del 'Nuevo Mundo' sólo es posible siendo fieles a una auténtica conciencia histórica de ser habitantes de un 'Nuevo Mundo'. Para ello hay que apropiarse de tal conciencia de manera radical y sin traicionarse a sí mismos. En relación con esto, hay que subrayar que para Mayz Vallenilla, el ser americano es un modo de ser histórico y como tal sólo puede revelarse en el transcurrir del tiempo y en la historia. De lo cual se sigue que los intentos programáticos de descubrir América o reconquistar un pasado que no le pertenece, para fijar allí la 'originariedad' de los latinoamericanos, son, en vez de originales, ilusorios y equivocados. Con base en esta crítica, Mayz Vallenilla los denuncia y hace un llamado para que los latinoamericanos dejen que Latinoamérica se manifieste, desde sí mismos y por sí misma, en su 'originariedad'. De esta manera, la experiencia del modo de ser latinoamericano se dará a través del tiempo en la historia, en vez de ser algo establecido programáticamente. Para él, esto no implica una suerte de quietismo ni una actitud meramente receptiva, sino, al contrario, una acción, intelectual y práctica, eminente y necesariamente creadora. La actitud que propone Mayz Vallenilla es la siguiente: "por ser americanos, ya en nuestro 'ser' nos está dada la comprensión original de América”. El Problema de América propone, pues, una hermenéutica existenciaria del ser americanos como hombres del 'Nuevo Mundo'. Esto se basa en que la búsqueda de una comprensión auténtica de América debe fundarse en el factum de que el 'ser' de los americanos tiene ya, en cada caso, una comprensión originaria de América en la que se halla implícita el sentido de ser nuevo - 'original'- del 'Nuevo Mundo'. 
Mayz Vallenilla comienza a tratar el tema de la ciencia y la técnica en Del Hombre y su Alienación, de 1966, donde confronta las concepciones de Marx y Heidegger sobre la alienación. Varios escritos preparatorios de una "Crítica de la Razón Técnica", con sus correspondientes nuevas categorías y principios, que Mayz enfrenta a la Crítica de la Razón Pura kantiana, constituyeron el Esbozo de una Crítica de la Razón Técnica, aparecido en 1974. El tema del afán de poder está estrechamente relacionado con el de la técnica. Mayz Vallenilla ve a dicho 'afán de poder como raíz de la técnica'. Dentro de esta línea de pensamiento, en 1982 apareció El Dominio del Poder, un esfuerzo por delinear una ética universal frente a los vicios y excesos del 'afán de poder', que resultan del uso incontrolado e incontrolable por parte del hombre de los instrumentos cada vez más complejos y potentes de la técnica. En la misma línea de preocupación intelectual está La Ratio Technica de 1983, trabajo en el cual se completa la crítica de la razón técnica. Una tesis de Mayz es que el 'logos técnico' es el principio ductor y organizativo de nuestro tiempo.

Llegamos finalmente a la obra fundamental de Ernesto Mayz Vallenilla, los Fundamentos de la Meta-Técnica, publicados en 1990. El término 'meta-técnica' fue acuñado en los años $80 \mathrm{y}$ utilizado por primera vez en el Congreso Mundial de Filosofía de Brighton. Con el mismo, Mayz Vallenilla designa una nueva modalidad de la técnica, en la cual se superan las características antropomórficas, antropocéntricas y geocéntricas que hasta ese momento habían prevalecido en la técnica tradicional, tanto en lo referente a su ars operandi, como en lo relativo a la índole y funciones de sus propios instrumentos. Ahora bien, de acuerdo con Mayz, esta superación de la noción tradicional de la técnica implica una transformación y transmutación radicales de la razón y la racionalidad que habían sustentado a la técnica como expresión del afán de poder del hombre.

Él piensa que desde un punto de vista filosófico, la razón y la racionalidad, desde sus inicios en los Presocráticos y Parménides, hasta Husserl, Heidegger y todos sus epígonos actuales, ha sido identificada (etimológica y filosóficamente) con un 'ver', ya sea dicho 'ver' de modalidad sensible y empírica o eidética e inteligible. De esto se sigue que la verdad racional sea en sí misma comprendida como expresión de una 'evidencia', el cual es un término de raigambre 'óptica lumínica' por su procedencia del latín videre, que significa 'ver', trátese de que tal verdad racional se funde en una evidencia empírica y/o intelectual. En tanto la razón es sinónima de un 'ver', para cumplir su función, ese 'ver' debe alimentarse y utilizar ingénitamente la 'luz visible', adueñándose, captando y procesando aquel tipo de ondas del espectro electromagnético que, por su longitud y frecuencia, se adaptan a las capacidades aprehensivas y receptoras del ojo humano como tal. Encontramos aquí uno de los aspectos primordiales que la Meta-técnica intenta superar frente a la técnica tradicional. Mayz Vallenilla apunta que en realidad ya lo ha hecho en nuestro tiempo. Con esto se refiere a la elaboración de nuevos instrumentos que son capaces de aprehender la alteridad y sus manifestaciones de una forma incomparablemente más amplia, rica y radicalmente diversa que la tradicional. La manera tradicional es, de acuerdo con él, óptica y lumínica, por lo tanto, estricta y limitadamente humana, tanto en sus fundamentos como en sus límites.

En cambio, hoy en día, se aprehende a la alteridad mediante otros instrumentos. Por ejemplo: mediante ondas sónicas o ultrasónicas, ya sea con el radar y el sonar, o mediante la ayuda de otros artefactos más complejos y sofisticados que proporcionan ecosonogramas de aquella alteridad; mediante estímulos térmicos, verbigracia, en los sensores utilizados para guiar misiles y proyectiles balísticos; mediante ondas de radio en los radio-telescopios; mediante rayos infrarrojos en satélites-telescopios precisamente diseñados para este fin; mediante resonancia magnética nuclear, en diversos instrumentos, como el SQUID (Superconducting Quantum Interference Device) que puede captar campos magnéticos utilizados para registrar el funcionamiento de las neuronas, la MRI (Magnetic Resonance Imaging) o la MRS (Magnetic Resonance Spectroscopy) que suministra el funcionamiento de la química cerebral en actividades tales como el aprendizaje, la memoria, las emociones y la propia visión; y otros muchos instrumentos. De acuerdo con la interpretación de Mayz, todos estos medios dan lugar a un nuevo logos meta-técnico, gracias al cual es posible ordenar y dar sintaxis al espacio y al tiempo, bases fundamentales de la organización de la alteridad, en virtud de códigos completamente diferentes a los tradicionales. La transformación radical que esto representa para los ordenamientos espacio-temporales de la alteridad afecta directamente a los conceptos y nociones epistemológicos y ontológicos de la tradición filosófica. Es decir, a conceptos como el ser y la nada, la afirmación y la negación, la conciencia y el conocimiento. También afecta y 
modifica las bases y fundamentos que constituyen supuestos tácitos en otras disciplinas esenciales, como la lógica, la geometría y las matemáticas. Con ello modifica la idea de naturaleza (physis) y la estructura y finalidades de las instituciones humanas fundadas sobre esas bases y nociones superadas por la meta-técnica, instituciones tales como el lenguaje, la ética y la política. Desde allí, las transformaciones se extienden hasta las bases y presupuestos de la biología, la antropología y la antropogonía (nacer/origen). Ernesto Mayz Vallenilla piensa que bajo el ámbito de la transformación que está produciendo la revolución meta-técnica, el propio ser humano puede ser sujeto de transformación o transmutación en su physis (naturaleza) ingénita y, por lo tanto, en sus congénitos límites somáticos y psicofísicos, con secuelas y consecuencias que no son difíciles de imaginar, en todos los sentidos, especialmente los axiológicos, como actualmente lo escuchamos y presenciamos entre las noticias del normal amanecer de cada día

Así pues, la razón y la racionalidad humanas, cuya genealogía y límites ópticolumínicos han dominado hasta nuestros propios días, se ven hoy radicalmente modificadas y sustituidas por un logos trans-óptico, trans-lumínico y trans-humano, diseñado y construido por el propio hombre con la ayuda de instrumentos técnicos que, a su vez, trascienden y modifican los límites antropomórficos, antropocéntricos y geocéntricos que ostentaban la razón y la racionalidad humanas tradicionales, tanto en lo relativo a sus principios como en sus categorías. Esta radical modificación, afecta y transmuta paralelamente tanto a los fundamentos de las ciencias, como a los de todas las instituciones creadas por el hombre con la ayuda de su innata razón y su correspondiente racionalidad tradicional, incluidos los fundamentos del lenguaje humano y sus sintaxis, como sustentáculos o bases de las creaciones, obras e instituciones humanas. Finalmente, Mayz Vallenilla señala que de esto surge la propuesta de una Nootecnia como disciplina y/o procedimiento traductor de la vieja a la nueva racionalidad y a sus correspondientes categorías, instrumentos y creaciones.

Bibliografía

MAYZ VALLENILLA, Ernesto: El Problema de la Nada en Kant, 2ª Edición, Caracas, Monte Ávila, 1992.

MAYZ VALLENILLA, Ernesto: El Problema de la Nada en Kant, $1^{\text {a }}$ Edición, Revista de Occidente, 1965. 